\title{
Tympanoplasty surgery: a retrospective audit of surgical outcomes at a regional hospital
}

\author{
S Naidoo, ${ }^{1}$ O Edkins, ${ }^{1}$ V Naran, ${ }^{1}$ S Liebenberg ${ }^{2}$ \\ ${ }^{1}$ University of Cape Town \\ ${ }^{2}$ Sessional Consultant, Department of Otorhinolaryngology, False Bay \& New Somerset Hospitals
}

Corresponding author: Shavina Naidoo (shavina.naidoo@gmail.com)

\begin{abstract}
Background: Chronic Suppurative Otitis Media (CSOM) is a common condition in our setting, resulting in tympanoplasty surgery being one of the common surgical procedures performed. Little is published regarding outcomes of tympanoplasty surgery in patients living with Human Immunodeficiency Virus (HIV), which has a high prevalence in South Africa.

Aims: To audit the outcomes of tympanoplasty surgeries performed, including outcomes in patients with HIV.

Methods: A retrospective review of all tympanoplasty surgeries performed at our institute. Outcomes of surgery according to patient demographics, documented HIV status, seniority of surgeon, type and techniques of graft placement and hearing outcomes were reviewed.

Results: Successful closure of perforations was $84.4 \%$. Closure rates in the 10 patients known to be HIV positive were $100 \%$. The number of patients meeting the criteria for The Belfast Rule of Thumb (BROT) increased from $26.3 \%(\mathrm{n}=20)$ preoperatively to $68.4 \%(n=52)$ postoperatively.

Conclusions: The results are comparable to both the results obtained at a tertiary hospital and those published internationally. Successful outcomes are obtainable in patients infected with HIV; however, as to the optimal timing of this surgery, this would require further investigation.
\end{abstract}

MeSH Keywords: Tympanoplasty, Belfast Rule of Thumb, Chronic Suppurative Otitis Media in HIV

S Afr J Surg 2018;56(3) http://dx.doi.org/10.17159/2078-5151/2018/v56n3a2363

\section{Introduction}

Chronic Suppurative Otitis media (CSOM) is a common presenting condition in our setting in sub-Saharan Africa, resulting in tympanoplasty surgery being one of the common surgical procedures performed in the department. Prevalence of CSOM in sub-Saharan Africa is between $0.4 \%$ and $4.2 \%$ with more than $50 \%$ of cases occurring under the age of 10 years. $^{1}$

Middle Ear Surgery has made many advances in the last century, with tympanoplasty being the main surgical treatment for CSOM. It was first described by Ludwig Wullstein in 1956, and today otologists have developed multiple techniques for graft placement and graft material that can be used. ${ }^{2}$ The basic principle behind tympanoplasty surgery is to eliminate or stabilise disease processes, to allow for easier access when doing postoperative care and, importantly for patients, to recondition the sound conduction mechanism within the middle ear. ${ }^{3}$

South Africa is a country with a high prevalence of Human Immunodeficiency Virus (HIV), with an estimated $10.2 \%$ of the total population being HIV positive. ${ }^{4}$ Another staggering statistic is that up to $100 \%$ of patients infected with HIV will at some point develop an ENT or head and neck manifestation of their disease, with a significant proportion affecting the health of the middle and inner ear. ${ }^{5}$ However, little is published regarding outcomes of tympanoplasty surgery in patients living with HIV. In our units this surgery is offered to HIV positive patients who are on antiretroviral medication and who have a suitable CD4 count $\left(>350\right.$ cells $\left./ \mathrm{mm}^{3}\right)$.

\section{Aims}

To audit the outcomes of tympanoplasty surgeries performed at New Somerset Hospital, Cape Town, a regional teaching hospital, and compare these results to internationally published data and the results of tertiary level units in our training platform. The outcomes to be audited would be the surgical closure rate of the perforated tympanic membrane with comparison of success rates of surgical techniques, graft material, experience level of surgeon and success rates in patients with a known HIV status, along with outcomes in hearing improvement after surgery.

A secondary objective was to assess the success of tympanoplasty surgery currently being performed at nontertiary hospitals within South Africa as both an expansion of the training platform for specialist registrars as well as a suggested revision on provincial policies relating to which procedures are suitable to be performed at the various levels 
of health care facilities, thus improving patient access to this surgery.

\section{Methods}

A retrospective folder review was conducted, looking at all tympanoplasty surgeries performed at our institute between July 2011 and February 2015. Ethics approval was obtained from the University of Cape Town Human Research and Ethics Committee.

Data collected was based on the objectives of the audit and included: patient demographics, pathology, HIV status (if documented), intraoperative findings (perforation size and ossicular chain integrity), surgical technique, grafting material, ossiculoplasty, experience level of surgeon, clinical findings at follow up visits, audiological status of the affected and contralateral ear pre- and postoperatively. Patient confidentiality was maintained and data was compiled and reviewed by the authors on a password-locked private database. Those included in the study needed to have had a pre- and postoperative audiogram and a follow-up at 3 months. The exclusion criteria for this study were inadequate documentation and patients that were lost to follow up without the necessary follow-up time ( $>3$ months) or postoperative audiograms having been done.

The definition of surgical closure was a morphologically intact tympanic membrane. Patient benefit and improvement of hearing were calculated using the Belfast Rule of Thumb (BROT) on audiograms before and after surgery, where the pure tone average (PTA) was taken to be equal to or better than $30 \mathrm{~dB}$ Hearing Level in the operated ear and within $15 \mathrm{~dB}$ difference of the contralateral ear (4) in order to meet the BROT. The change in PTA air conduction thresholds was assessed.

HIV status and CD4 counts that were documented at the preoperative visit were reviewed and no results were considered if they were documented after the surgery was performed. All HIV positive patients having surgery were already on antiretroviral therapy regimens. No patients were recalled to retest their status.

A comparison of prognostic factors as they relate to the primary outcomes was made using the Chi Square Test.

\section{Results}

A total of 159 patient records were reviewed who had undergone tympanoplasty surgery during the study period. Of these, 43 patients were lost to follow-up or had inadequate documentation for the purpose of the audit; 17 patients had clinical follow-up but had not returned for any postoperative audiometry assessment; 9 patients had returned for both their follow-up appointments and audiometry but were $<3$ months postoperative at the time of review of the records. These were all excluded from the study population. As a result, only a total of 90 patients were included in the study.

The demographics of the study population revealed a female to male ratio of $2.2: 1$, with the proportion of patients under the age of 18 years and those above 18 years of age at $42.2 \%$ and $57.8 \%$ respectively.

The total closure rate was found to be $84.4 \%$ with differing success rates for procedures performed by different levels of seniority, experience and supervision, in decreasing order respectively by consultants $(92.8 \%)$, registrars with a supervising consultant $(83.7 \%)$ and a registrar unsupervised $(81.5 \%)$. In the case of the registrar with supervising consultant group, the procedure would be supervised, or partly performed, by a consultant scrubbed in. There was however no statistical difference between the groups.

Overall hearing improvement showed an average (mean) improvement in PTA air conduction threshold of $10.4 \mathrm{~dB}$. Hearing improvement in cases of successful closure of the tympanic membrane showed an average $11.8 \mathrm{~dB}$ improvement in PTA air conduction thresholds. One patient who had successful closure had a reduction in hearing. Overall, consultants had the better improvements in hearing improvement from pre- to postoperatively when assessing the BROT criteria (Table 1). Overall the proportion of cases where patients fell within these criteria increased from $26.7 \%$ presurgery to $64.4 \%$ postsurgery. Out of the 76 patients with successful closure of the tympanic membrane, $61.8 \%$ $(\mathrm{n}=47)$ had $>10 \mathrm{~dB}$ improvement in PTA air conduction, with the number of patients being within the criteria of BROT increasing from $26.3 \%(\mathrm{n}=20)$ preoperatively to $68.4 \%$ $(\mathrm{n}=52)$ postoperatively. Of these patients who did not fit the criteria of BROT preoperatively, 7 patients had preoperative

Table 1. Comparison of tympanoplasty outcomes (all p-values $>0.05$ )

\begin{tabular}{|c|c|c|c|c|c|c|c|c|}
\hline & \multicolumn{2}{|c|}{ Registrar } & \multicolumn{2}{|c|}{ Registrar \& Consultant } & \multicolumn{2}{|c|}{ Consultant } & \multicolumn{2}{|c|}{ Total } \\
\hline & (n) & Percentage & (n) & Percentage & (n) & Percentage & (n) & Percentage \\
\hline Number of cases & 27 & $30.0 \%$ & 49 & $54.4 \%$ & 14 & $15,6 \%$ & 90 & - \\
\hline TM Closure & 22 & $81.5 \%$ & 41 & $83.7 \%$ & 13 & $92.8 \%$ & 76 & $84.4 \%$ \\
\hline $\begin{array}{l}\text { PTA BROT Pre } \\
\text { Surgery }\end{array}$ & 9 & $33.3 \%$ & 15 & $30.6 \%$ & 2 & $14.3 \%$ & 24 & $26.7 \%$ \\
\hline $\begin{array}{l}\text { PTA BROT Post } \\
\text { Surgery }\end{array}$ & 15 & $55.6 \%$ & 34 & $69.4 \%$ & 9 & $64.3 \%$ & 58 & $64.4 \%$ \\
\hline $\begin{array}{l}\text { PTA }>10 \mathrm{~dB} \\
\text { Improvement }\end{array}$ & 12 & $44.4 \%$ & 26 & $53.1 \%$ & 10 & $71.4 \%$ & 48 & $53 \%$ \\
\hline
\end{tabular}


hearing loss too severe to have any expected improvement from surgery, and surgery was performed for the purpose of obtaining a dry, disease-free ear. Thus out of the remainder of patients where functional hearing improvement was a possibility, 32 of the 49 patients $(65.3 \%)$ improved from a preoperative status of not meeting the BROT criteria to meeting these criteria postoperatively.

Comparison of graft material and placement resulted in very small numbers within each category, and were therefore not statistically significant. Overall cartilage had the better closure rates $(93.8 \%)$ when compared to temporalis fascia (TF) $(82.4 \%)$ as a graft material, however, with the numbers in the review, it was not shown to be statistically significant ( $p$-value $>0.05)$. Comparison of closure rates for various grafting techniques and material resulted in small numbers within each category thus not showing any statistically significant difference. The results were: TF underlay $84.6 \%$ $(\mathrm{n}=52)$, TF underlay enveloped around handle of malleus $80.0 \%(\mathrm{n}=10)$, TF underlay with anterior hammock $75.0 \%$ $(\mathrm{n}=12)$, cartilage butterfly $66.7 \%(\mathrm{n}=3)$, cartilage underlay $100 \%(n=10)$ and cartilage shield grafting $100 \%(n=3)$.

Just over a third of the study population was aware of their HIV status, with $64.4 \%$ falling into the unknown category. Ten patients (11.1\% of the study group) were known to be HIV positive, all of whom were on antiretroviral therapy and had CD4 counts above 350 cells $/ \mathrm{mm}^{3}$. The outcomes for patients, according to their HIV status, showed a closure rate of $100 \%$ $(\mathrm{n}=10)$ in those known to be HIV positive, $81.8 \%(\mathrm{n}=22)$ in the known HIV negative group and $82.8 \%(n=58)$ in those patients whose HIV status was documented as unknown.

Documented ossicular pathology or erosion was found to be present in $50 \%$ of the patients known to be HIV positive compared to $22.5 \%$ in the rest of the cases. Hearing outcomes postsurgery are outlined in Figure 1, showing that $55.6 \%$ of patients with HIV improved from a presurgery hearing status falling out of criteria of BROT to meeting those criteria postoperatively, when compared to the $54.3 \%$ finding in the HIV negative and unknown groups.

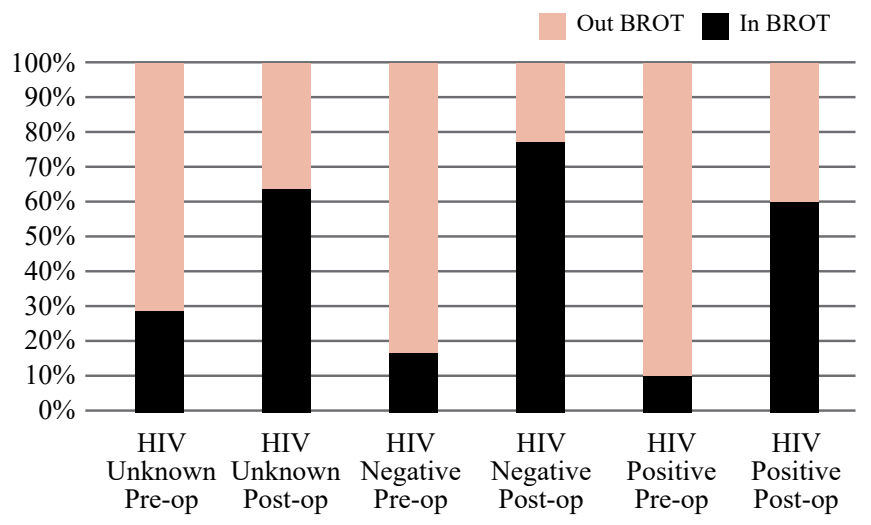

Figure 1. Pre- and postoperative hearing status of operated ears according to HIV status ( $p$-values $>0.05$ )

\section{Discussion}

Traditionally, technical success of surgery is measured based on closure rates of perforations, a healthy middle ear and reducing the air-bone gap, the latter being the improvement gain in hearing which is measured using audiometry. ${ }^{6}$ Although a very effective way of establishing surgical outcomes, it may not establish whether there has been a functional improvement of hearing, as appreciated by patients undergoing the surgery. This may require a different approach where the audiometric status of the opposite, non-operated ear is taken into account, especially where there is bilateral disease. ${ }^{6}$ The Belfast Rule of Thumb is such method, where subjective binaural hearing improvement is measured by assessing objective audiometric symmetry and the final hearing measurement in the better hearing ear. ${ }^{6}$

The main limitation to highlight in this retrospective study is that it did produce relatively small numbers, and most cases of exclusion were due to inadequacies in follow-up. This may be related to the large geographical area the hospital serves as well as various socio-economic factors that may negatively impact the health-seeking behaviour of patients. Inadequate documentation played a small role here.

Closure rates in this study matched those found in published international literature, along with similar data on success rates for graft material, temporalis muscle fascia versus cartilage grafts. Studies report success rates ranging from $82.9-84.3 \%$ for temporalis muscle fascia and $92-92.4 \%$ for cartilage grafts. ${ }^{7-8}$ In comparison to our neighboring tertiary training hospital, our overall outcomes showed adequately comparable results. Reported success closure rates were $71 \%$ with an average PTA improvement of $12.4 \mathrm{~dB}$ in air conduction postoperatively and $64 \%$ of patients had PTA air conduction thresholds better than $30 \mathrm{~dB}$ in the operated ear postoperatively. ${ }^{9}$ A significant proportion of patients had acceptable and recognisable improvement in hearing.

Outcomes for various grafting techniques and materials did not reveal any statistically significant results ( $p$-values $>0.05$ ) due to small numbers in each category and more than likely reflects the preference of the surgeon, type of pathology and the individual indication for the material chosen.

Significant portions of the population we serve are HIV positive, with 1 in 10 patients being affected. ${ }^{4,10}$ The percentage of patients known to be HIV positive in this study mirrors that of the reported South African prevalence. However, because not every patient included in the study was tested prior to surgery, the study population prevalence of HIV may be under-reported. There is little evidence to determine if this disease has any negative effects on the successful outcomes of tympanoplasty surgery. As a result, most policies in place are that of expert opinion, rather than evidence-based. Thus, our current approach is such that patients with HIV are on antiretroviral therapy and have a CD4 count of $350 \mathrm{cell} / \mathrm{mm}^{3}$ or better, prior to performing a tympanoplasty. The assumption is that the immune compromised state would be controlled, thus decreasing the chance of concurrent upper respiratory and middle ear pathology that may negatively influence the 
outcome of the surgery. The results of this study seem to show that in these cases there is no evidence to suggest that the outcomes are worse with our current practice; in fact they had a $100 \%$ closure rate. However, despite the small numbers and a $\mathrm{p}$ value $>0.05$ showing no statistical difference, it remains that there is no difference.

The study also revealed that of the HIV positive group a larger proportion of cases had documented ossicular disease, or erosion, when compared to the rest $(50 \%$ compared to $22.5 \%$ ), with only $10 \%$ of patients falling within the criteria for BROT preoperatively compared to $28.8 \%$ in the rest. Postoperative hearing outcomes showed the biggest improvement in hearing in the HIV positive group with similar outcomes in all categories of HIV status postoperatively. There was no statistical difference.

\section{Conclusion}

This review of our surgical outcomes shows that the results obtainable at a non-tertiary hospital are comparable to both the results obtained at a tertiary hospital in the same geographical area, as well as those published internationally. It is thus feasible, given the availability of the correct expertise and equipment, for the expansion of this type of surgical service and training platform to district and regional hospitals within South Africa. This study has also promisingly shown that successful outcomes are obtainable in patients infected with HIV suffering from CSOM. However, as to the optimal timing of the surgery in this group with regards to antiretroviral therapy, CD4 counts and possibly viral load, the development of a standardised policy or guideline would require further investigation in the form of randomised control trials.

\section{Summary:}

- Tympanoplasty surgery can be done successfully outside tertiary institutes, if the right level of expertise is available
- Training platforms for specialist registrars in performing this procedure may be expanded to non-tertiary units

- Successfully comparable outcomes of tympanoplasty are achievable in HIV positive patients, who are on antiretroviral therapy with adequate CD4 counts

- Good functional hearing outcomes are obtainable in the majority of cases

\section{REFERENCES}

1. Tiedt NJ, Butler IR, Hallbauer UM, Atkins MD, Elliott E, Pieters M, Joubert G, Seedat RY. Paediatric chronic suppurative otitis media in the Free State Province: clinical and audiological features. S Afr Med J. 16 May 2013;103(7):467-70.

2. Mohamad SH, Khan I, Hussain SS. Is Cartilage Tympanoplasty More Effective Than Fascia Tympanoplasty? Otology \& Neurology. 2012;33:699-705.

3. Müller J, Schön F, Brill S, Helms J, Hagen R, Würzburg S. "Germany Tympanoplasty today - an analysis of 11000 cases of reconstructive middle ear surgery - The Würzburg Experience, 4th International Symposium on Middle Ear Mechanics in Research and Otology MEMRO; 27-30 July 2006; Zurich.

4. Statistics SA. Mid-year Population Estimates 2014.

5. Tami TA, Hairston JA. HIV and Otolaryngology. In: Gleeson M, ed. Scott-Brown's Otorhinolaryngology, Head and Neck Surgery. London: Hodder Arnold, 2008; p. 238-50.

6. Nia JK, Bance M, Missiha S. Review of Methods Used to Estimate Patient Benefit from Middle Ear Surgery to Correct Unilateral and Asymmetrical Conductive Hearing Loss. Univ Toronto Med J. Mar 2001;78(2):114-6.

7. Iacovou E, Vlastarakos PV, Papacharalampous G, Kyrodimos E, Nikolopoulos TP. Is cartilage better than temporalis muscle fascia in type I tympanoplasty? Implications for current surgical practice. Eur Arch Otorhinolaryngol. Nov 2013;270(11):2803-13.

8. Demirci S, Tuzuner A, Karadas H, Ackkgoz C, Caylan R, Samim EE. Comparison of temporal muscle fascia and cartilage grafts in pediatric tympanoplasties. Am J Otolaryngol. Nov-Dec 2014;35(6):796-9.

9. Becker J, Lubbe D. Success rate of myringoplasty at Groote Schuur Hospital. S Afr Med J. 27 Sep 2011;101(10):740.

10. Lubbe DE. HIV and ENT. CME. 2004;22:250-3. 